\title{
Performance of photoelectron spin polarimeters with continuous and pulsed sources: from Storage Rings to Free Electron Lasers.
}

\author{
T. Pincelli,${ }^{a, b *}$ F. Grasselli, ${ }^{c, d}$ V. N. Petrov,${ }^{e}$ P. Torelli ${ }^{b}$ And G. Rossi ${ }^{a}, b$ \\ ${ }^{a}$ Dipartimento di Fisica, Università degli studi di Milano, Via Celoria 16, 20133, Milano, Italy, \\ ${ }^{b}$ Laboratorio TASC, IOM-CNR, S.S. $14 \mathrm{~km} \mathrm{163.5,} \mathrm{Basovizza,} \mathrm{34149,} \mathrm{Trieste,} \mathrm{Italy,}{ }^{c}$ Dipartimento di \\ Scienze Fisiche, Informatiche e Matematiche, Università degli Studi di Modena e Reggio Emilia, Via \\ Campi 213/a, Modena, Italy, ${ }^{d}$ S3, Consiglio Nazionale delle Ricerche, Istituto Nanoscienze, Via Campi \\ 213/a, Modena, Italy, and ${ }^{e}$ Saint Petersburg State Polytechnical University, Politechnicheskaya Street 29, \\ 195251, Russian Federation.E-mail: pincelli@iom.cmr.it
}

\begin{abstract}
In this work we theoretically derive the experimental uncertainties concerning electron spin polarization (SP) in various realistic measurement conditions. The accuracy on the evaluation of SP of the photoelectron current is analysed as a function of the detector parameters and specifications, as well as of the characteristics of the photoexcitation sources. In particular we have addressed the different behaviour of single counter or twin counter detectors when the intensity fluctuations of the source are considered, leading to a new definition of the SP detector performance. The widely used parameter called figure-of-merit (FOM) is shown to be inadequate to describe the efficiency of SP polarimeters, especially when they are operated with timestructured excitation sources such as Free Electron Lasers. Numerical simulations have been performed and yield strong implications in the choice of the detecting instruments in spin-polarization experiments, that are constrained in a limited measurement time. We therefore apply our results to the characteristics of a wide set of state-of-the-art spectroscopy facilities all over the world, and derive an efficiency diagram for SP experiments. Our results also define new mathematical instruments for handling the correct statistics of SP measurements in presence of the source intensity fluctuations.
\end{abstract}




\section{Introduction}

The measurement of spin polarization of electron beams is a long standing research programme that has led to the development of advanced instrumentation applied to diverse physical measurements, from nuclear decays to surface spectroscopy and magnetometry. As it is not possible to separate electrons on account of their spin state with the use of a Stern-Gerlach apparatus (Kessler, 1985), little efficient spin-dependent scattering effects, have to be exploited.

The scattering spin asymmetry can be produced by either exchange interaction between the electrons of the primary beam and the electronic cloud surrounding the target atoms, or by spin-orbit (LS) effects in the deflection electron beam by nuclei of a target. After a long history of developments (Getzlaff et al., 1998; Gay et al., 1992) of spin polarimetry based on LS asymmetry, the present state-of-the-art instruments exploit both kinds of interaction and can be divided in three classes:

1. High energy (0.05-0.5 MeV) LS scattering (Mott scattering) polarimeters, of which the Compact Classical Mott detector (Petrov et al., 2003), and the Rice-type Mott detector (Burnett et al., 1994) are the most popular designs. These detectors have low values of figure of merit (FOM), due to the small elastic back-scattering cross section of high energy electrons. However, target performance does not degrade on the scale of years, very long integration times are possible, and, if the target is a thin, highly transparent film, the single scattering approximation allows the polarimeter to be selfcalibrating. The asymmetry is measured as an imbalance in the count rate of two electron counters defining specular geometries and operated simultaneously.

2. Low energy LS scattering (Spin Polarized Low Energy Electron Diffraction - SPLEED), of which the Iridium Spin Filter (Kutnyakhov et al., 2013a) is the most recent application. Exploiting the Bragg beam formation in the LEED process, these instruments have higher FOM. They are especially suited for imaging systems, as they allow massive multichannel acquisition. The absence of applied magnetic fields enables to achieve spin-filtering without perturbing the electron spatial distribution. Target surface can be made stable (Kirschner et al., 2013). The asymmetry signal can be retrieved by combining the images observed at two different scattering electron energies.

3. Low energy exchange scattering (Very Low Energy Electron Diffraction - VLEED) of which the $\mathrm{Fe}(001)-\mathrm{p}(1 \times 1) \mathrm{O}($ Bertacco et al., 1998) is now facing a widespread success. These targets have almost two orders of magnitude larger FOM with respect to high energy LS polarimeters. Thanks to the use of the diffracted (00) beam and the high spin filtering efficiency of exchange scattering, they possess a 
large statistical advantage over instruments based on different scattering mechanisms. The passivated target has a reasonable stability in ultra-high vacuum (few weeks). The asymmetry is measured by combining subsequent signals recorded with opposite target magnetizations (Okuda et al., 2011).

As SP evaluation is the result of an asymmetry between two values of electron counts (or electron fluxes), a second distinction allows to categorize further these apparata, based on the measurement routine:

- Twin electron counters detectors (2CD), in which the asymmetry is obtained from the difference between two simultaneously measured signals from the electron counters (in the two specular geometries of the scattering events);

- One electron counter detectors (1CD), in which case asymmetry is evaluated by measuring the signal from a single detector, sequentially changing one of the scattering geometry parameters and measuring again.

Most of the instruments belonging to the low energy scattering classes (2. and 3.) are 1CDs, while the ones in the high energy scattering class (1.) are mostly 2CDs.

One further dimension to be considered is that each scattering geometry allows for the determination of the spin asymmetry along one of the vector components. If the full SP vector is to be measured then the three components must be obtained. Here it must be noted that two orthogonal components can be simultaneously measured with the high energy LS Mott polarimeters, using two couples of twin detectors at right angles that are independently but simultaneously sensitive to the two transverse spin polarization components of the incoming electron beam. The same result for a $1 \mathrm{CD}$ polarimeter requires four subsequent measures each with a geometry determined by the scattering sample magnetization that must be set in the four directions along the two perpendicular quantization axes.

As the new generation of extremely brilliant coherent and ultra-shortly pulsed sources is coming on-line (Free Electron Lasers -or FELs- (Galayda et al., 2010; Toru Hara, 2012; AmannJ. et al., 2012; Allaria et al., 2012; Allaria et al., 2013; Ayvazyan, V. et al., 2006), Ultraviolet solid state lasers, gas Generation of High Harmonics -or HHG- (Lorek et al., 2014; Leitner et al., 2011)), new photoemission techniques can be developed with access to different regimes of excitation and pump-probe protocols. Applications to high resolution measurements allowing for the simultaneous measure of energy, momentum and spin polarization as in ARPES experiments (Dil, 2009; Hoesch et al., 2002) has developed recently enhancing substantially the efficiency of Spin-ARPES (Das et al., 2016; Suzuki et al., 2014; Okuda et al., 2011; Bigi, 2016) and, in fact, making high resolution "complete photoemission experiments" possible (Schönhense et al., 2015). 
Understanding the optimal experimental approach to the SP measures in these novel experiments is of primary importance, both for the best exploitation of the scarce beam-time at FELs/X-FELs and for the best statistical value of the data.

The performance of a spin detector is classically evaluated by trying to achieve the highest Shermann function (for formal definition see Sect. 2 eq. 1) and the highest Figure of Merit (FOM)(for formal definition see Sect. 2 eq. 4). Aim of this work is to show that, in the present complex panorama of different applications, these two parameters might be misleading, and a full statistical analysis, accounting for the whole process behind the electron spin polarization measurement, is necessary to identify the most performing instrument for a certain application. We carried out such evaluation with particular attention to the statistics of electron spin polarization measurements accounting for the intensity fluctuations of the sources. This enables us to identify the various regimes in which each kind of instrument is best performing, and to lay down a map of the ranges of applicability.

\section{Spin-orbit based detectors}

Spin-orbit based apparata retrieve asymmetry from the process known as Mott scattering (Mott, 1929; Mott, 1932), studied by Sir N. Mott in 1929. When an incoherent beam of high energy electrons (0.05-0.5 $\mathrm{MeV}$ ) impinges on an heavy atom target, it is diffused incoherently in every direction of space, mostly due to the charge (Coulomb) scattering. A spin-orbit (LS) component of the scattering potential is nevertheless present as, in the frame of the electron, the target nuclei are seen as rotating charges during the scattering deflection, and the sense of rotation depends on the approaching trajectory of the electron with respect to the fixed nuclei in the crystal (and laboratory) frame. The relative amplitude of the LS scattering potential (anisotropic) with respect to the Coulomb potential (isotropic) depends strongly on the final deflection angle for the elastically scattered electrons. At small deflection angles the LS effect is negligible and non measurable, but at some large angles it becomes relevant. It has been demonstrated that for deflection angles of $120^{\circ}$ the LS effect on the total scattering cross section is relatively large and measurable: it is then possible to determine a geometry dependent asymmetry that corresponds to a SP asymmetry of the primary beam in the direction perpendicular to the scattering plane, defined by the beam itself and the detector position in the laboratory frame. If the primary beam has a polarization vector $\mathbf{P}^{\mathbf{1}}$, the differential cross section at

\footnotetext{
${ }^{\mathbf{1}}$ The polarization vector $\mathbf{P}$ of an ensemble of electrons is defined as

$$
\mathbf{P}=\frac{\sum_{n}\left\langle\varphi^{(n)}|\boldsymbol{\sigma}| \varphi^{(n)}\right\rangle}{\sum_{n}\left\langle\varphi^{(n)} \mid \varphi^{(n)}\right\rangle}
$$
}


angle $\vartheta$ is given by:

$$
\left(\frac{d \sigma}{d \Omega}\right)_{S L}=I(\vartheta)[1+S(\vartheta) \mathbf{P} \cdot \hat{\mathbf{n}}]
$$

where:

- $I(\vartheta)$ is the spin-averaged cross-section, cancelling out the effects of spin;

- $S(\vartheta)$ is a function, called Sherman function (Sherman, 1956), dependent on the angle of deflection, that expresses the efficiency with which the scattering process selects the spin;

- $\hat{\mathbf{n}}$ is the quantization axis unit vector.

If a partially polarized beam (with non-vanishing components of $\mathbf{P}$ in the direction of $\hat{\mathbf{n}}$ ) impinges with an energy in the proper range on a heavy atom target, then, the number of electrons scattered on the right $\left(N(\vartheta)=N_{r}\right)$ and on the left $\left(N(-\vartheta)=N_{l}\right)$ will be different, and the asymmetry function can be defined:

$$
A(\vartheta)=\frac{N_{r}-N_{l}}{N_{l}+N_{r}}
$$

Therefore, by measuring $A(\vartheta)$, it is possible to evaluate the component normal to the scattering plane of the spin-polarization vector of the primary beam:

$$
P=\frac{1}{S(\vartheta)} A(\vartheta)
$$

This is the basic operational principle of Mott polarimeter (Kessler, 1985), that withstood a long process of evolution and refinement from the first prototypes (Shull et al., 1943) to the present advanced instruments (Petrov et al., 2007; Pincelli et al., 2016; Strocov et al., 2015). It is crucial to note, in the light of the following discussion, that these polarimeters intrinsically allow for the use of two electron counters operated simultaneously at opposing angles. In fact the geometry asymmetry (left-right) corresponding to the LS extra deflection can be exploited directly. A 1D Mott scattering experiment can certainly be done, by measuring subsequent intensities for electron beams of reversed SP, but is certainly not the efficient way to go!

In fact even four detectors can be operated in a Mott polarimeter as both the transversal components of the SP of the electron beam can be simultaneously determined along two mutually perpendicular scattering planes both containing the electron beam.

It must be appreciated that a scattering angle of $120^{\circ}$ is quite unfavourable at these energies (single scattering regime, thin target film to avoid multiple scattering) and that consequently the overall counting statistics is low.

where $\left|\varphi^{(n)}\right\rangle$ are the pure spin states and $\boldsymbol{\sigma}$ is the Pauli operator, i.e. a three component vector of Pauli matrices. It is therefore, along any spatial direction, the ensemble-averaged expectation value of the spin operator. Its modulus is therefore always between 0 (completely unpolarized beam) and 1 (beam completely polarized along one direction). 
When comparing different kinds of polarimeters, it is customary to use a quantity called figure of merit (FOM). It roughly quantifies the performance of a spin detector, and is defined as:

$$
\varepsilon=\left(\frac{N_{r}+N_{l}}{N_{0}}\right) S_{\text {eff }}^{2}
$$

where $N_{0}$ is the beam intensity entering the polarimeter, $N_{l}+N_{r}$ is the total scattered intensity measured by the detectors and $S_{\text {eff }}$ is the effective Sherman function ${ }^{2}$ of the detector. As it will be discussed below, state of the art Mott polarimeters based on high-energy spin orbit scattering can achieve $\varepsilon=6 \times 10^{-4}$, mainly due to the low value of the ratio $\left(N_{r}+N_{l}\right) / N_{0}$ : the cross section of such large angle deflections required to efficiently select spin is rather low.

Lower energy apparata based on spin-orbit effects have also been built, both as 2CD (Yu et al., 2007) and as 1CD (Tusche et al., 2013; Kutnyakhov et al., 2013b; Kirschner et al., 2013). From the purely theoretical point of view, low-energy 2CD systems can be described with the same Eq. 1, Eq. 2 and Eq. 3, obviously with a different effective $S(\vartheta)$ as the multiple scattering dominates at low electron energies and the $S(\vartheta)$ cannot be calculated simply as in the case of single scattering. The low-energy $1 \mathrm{CD}$ systems, recently developed, require that the energy of the primary beam is modified to evaluate the asymmetry: the discussion of the performance of these systems can be developed along the same lines of the one for exchange based detectors, described below.

\section{Exchange based detectors}

As the energy of the primary is lowered enough the electrons interact in the full multiple scattering regime with the detailed electronic structure of the solid. Exchange effects take the dominant role determining the SP dependent amplitudes. These experiments require the use of thin films of $3 \mathrm{~d}$ ferromagnets as a target, possessing a reduced spin-orbit scattering contribution but a large exchange splitting of the electronic states. The quantization axis is now given by the magnetization direction of the target, which defines the component of the polarization vector that is probed. In particular, Bertacco \& Ciccacci (1999) demonstrated that the exchange-split band-gap in the empty states $\left(6 \mathrm{eV}\right.$ above $\left.E_{f}\right)$ of the $\mathrm{Fe}-\mathrm{O}(1 \mathrm{x} 1) \mathrm{p}$ passivated surface gives a very strong asymmetry both in reflection and adsorption when a polarized beam impinges on it. Building

\footnotetext{
${ }^{2}$ While the Sherman function is calculated for an ideal, single atom scattering experiment, in a real detector other factors can arise that further reduce the efficiency of spin selection: contamination of the target, multiple elastic scattering, finite angular acceptance of the detectors. Furthermore, the scattering angle and energy are fixed to the values that maximize the asymmetry. The effective Sherman function will therefore be a number obtained by a calibration measurement, accounting for all such different effects.
} 
the formalism in analogy with Eq. 1:

$$
\left(\frac{d \sigma}{d \Omega}\right)_{e x}=I(\vartheta)\left[1+S^{(e x)}(\vartheta) \mathbf{P} \cdot \hat{\mathbf{m}}\right]
$$

where $\hat{\mathbf{m}}$ is a unit vector representing the direction of the target's magnetization. When building the normalized asymmetry (as in Eq. 2), however, the radical difference between the two approaches sets in. Having lost the LS dominant effect, the geometrical asymmetry is lost and with it the possibility of measuring simultaneously the right-left asymmetry, i.e. to perform two specular experiments with two detectors per each scattering plane. Exchange based detectors require that an intensity measurement is performed with magnetization in the "up" direction $\left(N_{\uparrow}\right)$, then magnetization is reversed in the "down" direction, and a second acquisition $\left(N_{\downarrow}\right)$ is made. These two can be combined to give a single evaluation of asymmetry:

$$
A_{e x}=\frac{N_{\uparrow}-N_{\downarrow}}{N_{\uparrow}+N_{\downarrow}}
$$

Through the cross section in Eq. 5 it is then possible to find:

$$
P_{m}=\frac{1}{S_{\text {eff }}^{(e x)}} A_{e x}
$$

where $P_{m}$ is the component of polarization in the magnetization direction and $S_{\text {eff }}^{(e x)}$ represents the effective Shermann function of the system.

It is now clear that, requiring two separated measurements and an energy so low (6 eV) that only the (00) beam of LEED pattern appears, exchange interaction based apparata are intrinsically $1 \mathrm{CD}$. These systems where developed in the quest for higher efficiencies with continuous and stable sources, and they actually represent an extraordinary advancement $\left(\varepsilon \approx 10^{-2}\right.$ have been reached (Graf et al., 2005)).

\section{Error in polarization measurement}

In order to discuss the topic more clearly, it is better to introduce the basic formulas by deriving them under the assumption of a non-fluctuating stationary electron current. This will also enable to show the conceptual pattern that is repeated in the following steps, when coping with the more complicated temporal fluctuation regime.

The uncertainty on polarization defined in Eq. 3 can be evaluated, using the propagation for independent errors, as:

$$
\Delta P=P \sqrt{\left(\frac{\Delta A}{A}\right)^{2}+\left(\frac{\Delta S_{\mathrm{eff}}}{S_{\mathrm{eff}}}\right)^{2}}
$$


$\Delta P$ has two sources: a statistical one (related to $\Delta A$ ), and a systematic one (caused by $\Delta S_{\text {eff }}$ ). The systematic error is reduced during the calibration of the detector, when $S_{\text {eff }}$ is measured as accurately as possible. However, it is very difficult to realize accurate measurements because of the intrinsic low statistics of both methods of calibration ${ }^{3}$. Once $\Delta S_{\text {eff }}$ is determined, anyway, it cannot be changed and does not affect significantly the measurement routine, as substitution of typical values show that even a $10 \%$ uncertainty on $S_{\text {eff }}$ does not contribute significantly to $\Delta P$. As it is irrelevant to our discussion, in the following it will be neglected. The only uncertainty on $P$ is therefore assumed to be due only to statistical counting error in $\Delta A$.

\footnotetext{
${ }^{\mathbf{3}}$ The first method is double scattering (Kessler, 1985). The second method is called energy acceptance reduction (Gay \& Dunning, 1992).
} 


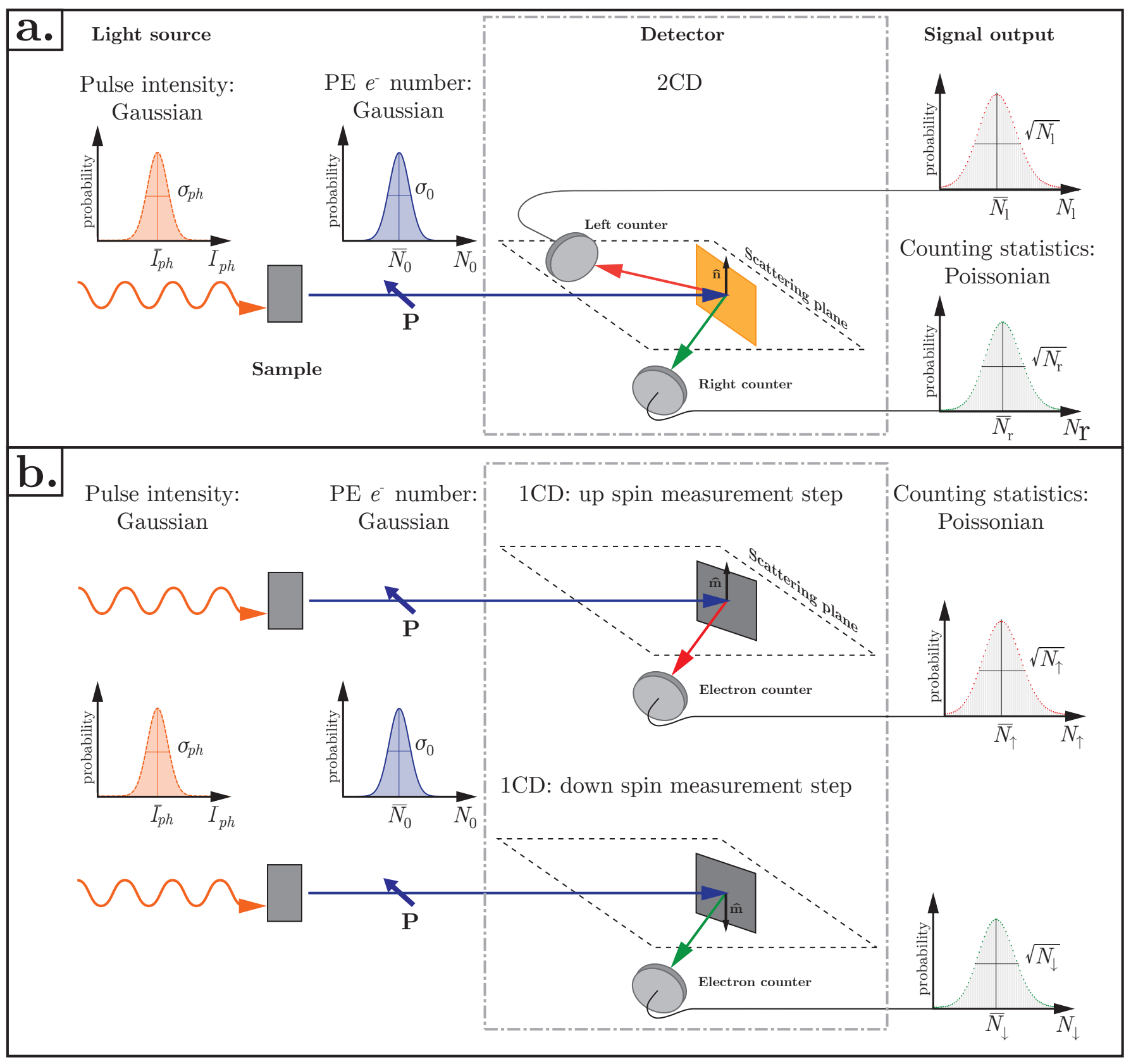

Fig. 1. a. Simplified scheme of the operation and of the accounted probability distributions in a 2CD detector. b. Simplified scheme of the operation and of the accounted probability distributions in a 1CD detector. It is divided in two subfigures in which the two successive steps needed for a single polarization measurement are shown.

The relative error $\delta A=\Delta A / A$ can be calculated as (see Eq. 2):

$$
\delta A=\sqrt{\left(\frac{\Delta \text { Diff }}{N_{r}-N_{l}}\right)^{2}+\left(\frac{\Delta \text { Sum }}{N_{r}+N_{l}}\right)^{2}}
$$

where $\Delta$ Diff $(\Delta$ Sum $)$ is the absolute error on $N_{r}-N_{l}\left(N_{r}+N_{l}\right)$. It follows that $\Delta$ Diff $=\Delta$ Sum. The second summand can be neglected, as it is much smaller than the first:

$$
\delta A \approx \sqrt{\left(\frac{\Delta \text { Diff }}{N_{r}-N_{l}}\right)^{2}} \equiv \sqrt{\frac{\left(\Delta N_{l}\right)^{2}+\left(\Delta N_{r}\right)^{2}}{\left(N_{r}-N_{l}\right)^{2}}}
$$


Assuming that the variances for $N_{r}$ and $N_{l}$ are determined by two independent Poissonians ${ }^{4}$, and:

$$
\Delta A=\delta A \cdot A=\frac{1}{\sqrt{N_{l}+N_{r}}}
$$

and, dividing by $S_{\text {eff }}$ to obtain polarization uncertainty:

$$
\Delta P=\sqrt{\frac{1}{\left(N_{r}+N_{l}\right) S_{\mathrm{eff}}^{2}}}
$$

now, introducing $N_{0}$ as defined in Eq. (4), it is possible to write:

$$
\varepsilon=\left(\frac{I}{I_{0}}\right) S_{\mathrm{eff}}^{2}=\left(\frac{N_{r}+N_{l}}{N_{0}}\right) S_{\mathrm{eff}}^{2}
$$

Finally, one has:

$$
\Delta P=\frac{1}{\sqrt{N_{0} \varepsilon}}
$$

For this reason the FOM has been regarded as a fundamental parameter of SP experimental apparata until now. However, as it will be shown in the following, the advent of pulsed sources with strong intensity fluctuations is dramatically changing the experimental conditions, reducing the importance of such parameter and shifting the focus towards set-ups able to reject the source intensity noise.

\section{Absolute error in presence of intensity fluctuations}

As discussed above, a measurement of electron beam SP by necessity consists in a difference between two measured intensities. The electrons are always counted in a finite, discrete time, that we will call a "measurement step": either the integration time window (with a continuous or quasi continuous source), or the duration of the bunch generated by a single excitation pulse as it is the case with the novel short pulsed sources. In both cases, the intensity fluctuations of the source, although on very different time-scales, must be thoroughly accounted to understand their role in the statistics of polarization measurement. In the following, the intensity fluctuations will be discussed in general. At the end of each chapter, the discussion will be exemplified for the case of measurement of the spin polarization of the secondary electrons, in an experiment in which separate, high intensity shots from a FEL are measured. This case represents the limit case, in which fluctuations are extremely large, while the intensity is extremely high. This is the region in the parameter space that yields the most counterintuitive results. At the end of the section, the whole parameter

\footnotetext{
${ }^{4}$ This is true since the signals are retrieved from two independent detectors.
} 
space will be addessed. The discussion (bearing no constraints on the actual timescale of measurement step) can therefore be adapted to all kind of sources.

In the following discussion we will assume that the electron detectors and counters of the polarimeter are able to handle the electron bunches scattered off the target surface without saturating. As the number of electrons reaching the detector can have very high peak currents (up to $10^{12} \mathrm{e} / \mathrm{s}$, corresponding to a bunch of $10^{5}$ electrons spread over about $100 \mathrm{~ns}$ ), this is not an obvious task. Very recently, however, it has been demonstrated in a state of the art Mott polarimeter set-up (Pincelli et al., 2016), so we considered it a solvable experimental problem and we neglected it.

\subsection{Fluctuations for $2 C D$}

The number of primary electrons is now defined by measurement step:

$$
N_{0}=N_{0}(i)
$$

where $N_{0}(i)$ is the number of electrons entering the detector during the $i$-th measurement step. It is possible ${ }^{\mathbf{5}}$ to assume that this is proportional to the intensity of the light during the during the $i$-th measurement step. The intensity distribution is generally described by a Gaussian with finite variance.

If a $2 \mathrm{CD}$ is considered, it is possible to treat the single measurement step in the same way as was done for a continuous flux of electrons. By simple substitution of $N_{0}$ in Eq. (14), we obtain:

$$
\Delta P(i)=\frac{1}{\sqrt{N_{0}(i) \varepsilon}}
$$

Performing a weighted average over $n_{p}$ measurement steps, the uncertainty reads:

$$
\Delta P\left(n_{p}\right)=\frac{1}{\sqrt{\sum_{i=1}^{n_{p}} \frac{1}{\Delta P(i)^{2}}}}=\frac{1}{\sqrt{\sum_{i=1}^{n_{p}} N_{0}(i) \varepsilon}}
$$

This procedure is thus very efficient in handling the instabilities of the source, because the weighted average enables to take little account of polarization measurements coming from very low intensity measurements that carry a very high uncertainty.

If one assumes to know the average number of electrons per measurement step, $N_{e p p}$, it is possible to consider $n_{p}$ so big that:

$$
\sum_{i=1}^{n_{p}} N_{0}(i)=n_{p} \bar{N}_{e p p}
$$

\footnotetext{
${ }^{\mathbf{5}}$ The effects of space charge, that distort the proportionality between light intensity and electron yield (Fognini et al., 2014), are neglected here.

IUCr macros version 2.1.10: 2016/01/28
} 
and to consider the asymptotic behaviour:

$$
\Delta P\left(n_{p}\right) \approx \sqrt{\frac{1}{n_{p} \bar{N}_{e p p} \varepsilon}}
$$

The uncertainty thus scales as the square root of $n_{p}$.

If we include the typical values of measurement of the spin polarization of the secondary electrons, e.g.

(Petrov \& Kamochkin, 2004; Fognini et al., 2014) $\varepsilon \approx 6 \times 10^{-4}$ and $\bar{N}_{e p p} \approx 10^{5}$, we obtain:

$$
\Delta P\left(n_{p}\right) \approx \frac{0.129}{\sqrt{n_{p}}}
$$

\subsection{Fluctuations for $1 C D$}

The asymmetry for a 1CD polarimeter was given in Eq. (6) but a subtle point must be considered when passing to the fluctuation regime. If the intensity counted in each measurement step was constant and equal to $\mathcal{N}_{0}$, the asymmetry that one would expect to measure is:

$$
A^{t h}=\frac{\mathcal{N}_{0} \sigma_{\uparrow}-\mathcal{N}_{0} \sigma_{\downarrow}}{\mathcal{N}_{0} \sigma_{\uparrow}+\mathcal{N}_{0} \sigma_{\downarrow}}
$$

where $\sigma_{\uparrow}$ and $\sigma_{\downarrow}$ are the cross sections for electrons with opposite spin state. In the real measurement, however, the electrons for $\sigma_{\uparrow}$ and $\sigma_{\downarrow}$ are subject to changes due to source fluctuations, and their number is different. It is then necessary to write:

$$
A^{r e a l}(i, i+1)=\frac{N_{\uparrow}(i)-N_{\downarrow}(i+1)}{N_{\uparrow}(i)+N_{\downarrow}(i+1)}
$$

Each of the measurements of $N_{\uparrow(\downarrow)}$ will be affected by the usual counting statistics of the electron counters so that:

$$
N_{\uparrow}(i)=\left(N_{0}(i) \sigma_{\uparrow}\right) \pm \Delta N_{\uparrow s t a t}(i)
$$

and

$$
N_{\downarrow}(i+1)=\left(N_{0}(i+1) \sigma_{\downarrow}\right) \pm \Delta N_{\downarrow s t a t}(i+1)
$$

If one wants to refer to the expected asymmetry, however, one must introduce a third error "pairwise" $\Delta N_{\mathrm{pp}}$ when computing the numerator and denominator, due to the fact that intensity fluctuations exist from one measurement step to the next ${ }^{\mathbf{6}}$. It is then possible to calculate the relative uncertainty:

$$
\delta A(i, i+1)=\sqrt{\frac{\Delta N_{\uparrow s t a t}^{2}(i)+\Delta N_{\downarrow \text { stat }}^{2}(i+1)}{\left[N_{\uparrow}(i)-N_{\downarrow}(i+1)\right]^{2}}+\frac{\Delta N_{\mathrm{pp}}^{2}(i, i+1)}{\left[N_{\uparrow}(i)-N_{\downarrow}(i+1)\right]^{2}}}
$$

\footnotetext{
${ }^{6}$ It must be noted that the errors are of a statistical nature. Despite the fact that now $\Delta N_{\mathrm{pp}}$ is introduced as a difference between two precise measurement steps, if one wants to deal with it as an error on the intensity measurement, one must consider $\Delta N_{\mathrm{pp}}$ as the variance of the distribution of the differences, just as one uses the variance of the Poissonian distribution centred at $N_{\uparrow}(i)$ for $\Delta N_{\uparrow s t a t}(i)$ or at $N_{\downarrow}(i+1)$ for $\Delta N_{\downarrow s t a t}(i+1)$.

IUCr macros version 2.1.10: 2016/01/28
} 
where $\left[\Delta N_{\uparrow s t a t}^{2}(i)+\Delta N_{\downarrow s t a t}^{2}(i+1)\right]$ is the absolute error on the numerator of $A$ and the relative errors concerning the denominator have been neglected. Considering Poisson statistics for the counting errors, it is now possible to obtain the absolute error on asymmetry and, dividing by the effective Shermann function $S_{\text {eff }}$, the absolute error on polarization:

$$
\Delta P(i, i+1)=\frac{1}{S_{\mathrm{eff}} \sqrt{N_{\uparrow}(i)+N_{\downarrow}(i+1)}} \sqrt{1+\frac{\Delta N_{\mathrm{pp}}^{2}}{N_{\uparrow}(i)+N_{\downarrow}(i+1)}}
$$

it is then evident that an object very close to the uncertainty observed in 2CDs is obtained by multiplication of the pre-factor with the first summand under the square root.A second term, arising from the instability of the source, is now present and increases the error as there are only non-negative numbers.

If several measurements are performed, a weighted average can be used again, yielding:

$$
\Delta P\left(n_{p}\right)=\frac{1}{\sqrt{\sum_{i=1}^{n_{p}} \frac{S_{\mathrm{eff}}^{2}\left[N_{\uparrow}(i)+N_{\downarrow}(i+1)\right]^{2}}{N_{\uparrow}(i)+N_{\downarrow}(i+1)+\Delta N_{\mathrm{pp}}^{2}} \delta_{(i \bmod 2,1)}}}
$$

where $\delta_{(i \bmod 2,1)}$ is the Kronecker Delta and mod is the modulo function, allowing to keep only the odd values of $i$. This is because each asymmetry value is now obtained only after two measurement steps. Stepping again to the limit of large $n_{p}$ it is possible to consider:

$$
S_{\text {eff }}^{2}\left[N_{\uparrow}(i)+N_{\downarrow}(i+1)\right] \approx S_{\text {eff }}^{2}\left(\bar{N}_{\uparrow}+\bar{N}_{\downarrow}\right)=2 \bar{N}_{0} \varepsilon
$$

the sum results in a simple multiplication by the number of observed asymmetry values i.e. $n_{p} / 2$, giving:

$$
\Delta P\left(n_{p}\right)=\sqrt{\frac{1}{n_{p} \bar{N}_{0} \varepsilon}\left(1+\frac{\Delta N_{\mathrm{pp}}^{2}}{\bar{N}_{\uparrow}+\bar{N}_{\downarrow}}\right)}
$$

Where $\bar{N}_{\uparrow}$ and $\bar{N}_{\downarrow}$ are the average intensities measured in the two magnetization configurations, $\bar{N}_{0}$ is the average number of electrons per measurement step before the polarimeter, and $\varepsilon$ is the FOM of the detector given in Eq. (4), substituting $N_{r(l)}$ with $\bar{N}_{\uparrow(\downarrow)}$.

To evaluate $\Delta N_{\mathrm{pp}}$ the discussion needs to be deepened slightly further. This uncertainty is generated by the fact that $N_{0}(i)$ and $N_{0}(i+1)$ are two successively and independently extracted variables from the same normal distribution:

$$
\mathcal{P}\left(N_{0}(i)\right)=\frac{1}{\sqrt{2 \pi \sigma_{0}^{2}}} e^{-\frac{\left(N_{0}(i)-\bar{N}_{0}\right)^{2}}{2 \sigma_{0}^{2}}}
$$

Where $\sigma_{0}$ is the variance in the distribution of number of primary electrons. This quantity can be traced back to the relative variance $\gamma \equiv \sigma_{p h} / \bar{I}_{p h}=\sigma_{0} / \bar{N}_{0}$ in the photon beam intensity on the appropriate time-scale, i.e. the same of the measurement step. The latter equality comes from the relationship between intensity IUCr macros version 2.1.10: 2016/01/28 
and number of photoemitted electrons that is supposed to be linear in this range. $\Delta N_{\text {pp }}$ can therefore be calculated by Gaussian integration (see Supplementary materials, Sect. I), giving:

$$
\Delta N_{\mathrm{pp}} \approx \frac{1}{\sqrt{2}}\left(\bar{N}_{\uparrow}+\bar{N}_{\downarrow}\right) \gamma \approx \frac{\sqrt{2} \bar{N}_{0} \varepsilon}{S_{\mathrm{eff}}^{2}} \gamma
$$

Where the last approximation comes from Eq. 27. Substituting in Eq. 28 we obtain:

$$
\Delta P\left(n_{p}\right) \approx \sqrt{\frac{1}{n_{p}} \cdot\left[\frac{1}{\bar{N}_{0} \varepsilon}+\frac{\gamma^{2}}{S_{\mathrm{eff}}^{2}}\right]}
$$

in the regime of large $\bar{N}_{0}$, the first summand of the factor in square parenthesis is much smaller than the other. It is then possible to write:

$$
\Delta P\left(n_{p}\right) \approx \sqrt{\frac{1}{n_{p}}} \cdot \frac{\gamma}{S_{\mathrm{eff}}}
$$

Applying again to the case of the spin-polarization of the secondary electrons with FEL source, we can substitute $^{7}: S_{\text {eff }} \approx 0.35$ (Okudaet al., 2011) $\bar{N}_{0} \approx 10^{5} \gamma \approx 0.1$ then one obtains:

$$
\Delta P\left(n_{p}\right) \approx \frac{0.286}{\sqrt{n_{p}}}
$$

i.e. despite the lower FOM, the 2CDs have a coefficient a factor of 3 smaller. This means that a repetition rate 9 times smaller is required to perform experiments with the same precision and the same duration, or that 9 times shorter experiments are required to $2 \mathrm{CDs}$ at the same repetition rate. It is interesting to observe that, in the parameter configuration used in this example, the coefficient of the $1 \mathrm{CD}$ is strongly dependent on $\gamma$, and only weakly on $\bar{N}_{0}$ (Eq. 31), while for 2CD the situation is exactly opposite: the coefficient is determined mainly by $\bar{N}_{0}$ and has no dependence on $\gamma$.

Our derivation therefore allows to individuate some regimes in which the advantage of monitoring the intensity given by $2 \mathrm{CD}$ s results in shorter measurements despite the higher efficiency attainable with $1 \mathrm{CDs}$. In Fig. 2, a density plot of the difference between the absolute error values of $1 \mathrm{CDs}$ and $2 \mathrm{CDs}\left(\Delta P_{1 C D}-\Delta P_{2 C D}\right)$, for a 100 measurement steps average, is plotted versus the number of electrons entering the detector per measurement step and of the intensity fluctuations of the source.

As our paper aims at mapping out the regimes (and consequently the cathegory of lightsources) with which the measurement mechanism of every spin detector is most efficiently used, we overlaid to the density plot a scheme depicting the regime of operation of state-of-the-art lightsources.

As explained in Sect. 5, it is also possible to account for intensity fluctuations also for continuous sources. If the R.M.S. fluctuations are evaluated on the same time window as the integration time of the electron

\footnotetext{
${ }^{7}$ It should be noted that $\gamma \approx 0.1$ is a rather generous estimate, based on the reported (Toru Hara, 2012) performance of SACLA FEL in Japan. Most FELS operate with higher shot-to-shot fluctuations.

IUCr macros version 2.1.10: 2016/01/28
} 
counters, the statistical treatment of both $1 \mathrm{CD}$ and $2 \mathrm{CD}$ errors is identical to the one followed above. For this reason, continuous and quasi-continuous sources have been added to Fig. 2. For continuous sources, the time duration of a measurement step was arbitrarily set at one second, and correspondingly the r.m.s intensity fluctuations on one second gating time were used.

Estimate of photoemission experiment intensities have been obtained:

- by using measured data when available

- by multiplying the photon flux and its R.M.S. fluctuations, assuming

- Quantum Efficiency (Q.E.) of a Gold Surface, $10^{-1} @ 30 \mathrm{eV}, 10^{-3} @ 6-7 \mathrm{eV}$,

$-10^{-3}$ in case of direct trasport to spin detector, $10^{-5}$ for detection after energy analyzer.

Considering the following issues as solvable by adapting the individual apparatus to the effective mode of operation, we have ignored the following:

- space charge effects,

- fine details of electrostatic lenses transmittance,

- the possibility of saturation of electron counters,

- sample degradation effects or disruption by coulomb explosion.

Care must be taken in reading this diagram, as the effective time required for an experiment is also defined by the repetition rate of the source, which is somehow hindered by the normalization to the number of measurement step used here: $\mathrm{J}$ operates at $20 \mathrm{kHz}$ for example, while $\mathrm{G}$ at $10 \mathrm{~Hz}$. The detailed source parameters are listed in Supplementary Materials, Sect. II. 


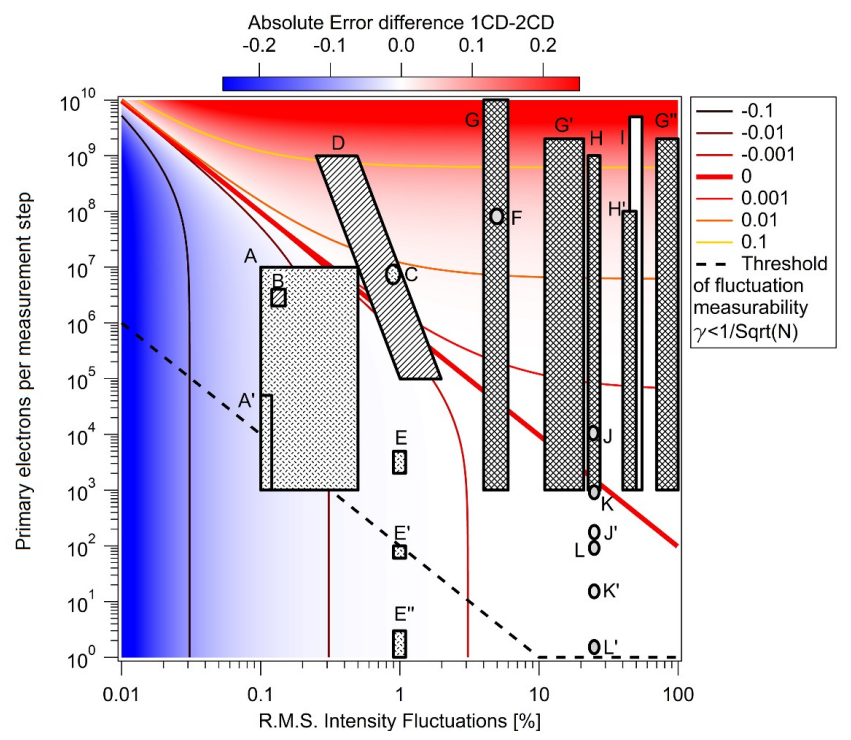

Fig. 2. Expected range of application with different sources. The dotted mesh (A,A',C,E,E',E") defines quasi-continuous sources, the continuous line mesh defines continuous sources (B,D), light gray mesh defines pulsed solid state lasers (F,J,J',K,K',L,L'), the rombohedral mesh "hot" FELs (G,G',G",H,H') and the white fill indicates superconducting FELs (I). A,A' - Synchrotron Beamline. B - Helium Lamp . C - quasi-CW VUV Laser. D - Electron gun. E',E" - Femtoslicing synchrotron beamline. F - Table-top laser 4HG. G - LCLS Hard-X FEL. G' - Sacla Hard-X FEL. G" - LCLS Self-seeding. H - Fermi FEL-1 (Seeded). H' - Fermi FEL-2 (Seeded,cascaded). I - FLASH FEL (SASE). J,K,J',K' - High power HHG Laser (Fluct. inferred from L). L,L' - Mid-power HHG Laser (for which shot-to-shot fluctuations were accurately measured).

\begin{tabular}{|c|c|c|c|}
\hline Symbol & Reference & Symbol & Reference \\
\hline $\mathrm{A}, \mathrm{A}^{\prime}$ & $\mathrm{A}^{\dagger}$ & $G^{\prime}$ & (Toru Hara, 2012) \\
\hline B & (Okuda et al., 2011) & G" & (AmannJ. et al., 2012) \\
\hline $\mathrm{C}$ & (Shimojima et al., 2015) & $\mathrm{H}$ & (Allaria et al., 2012) \\
\hline $\mathrm{D}$ & $\mathrm{D}^{\ddagger}$ & $H^{\prime}$ & (Allaria et al., 2013) \\
\hline $\mathrm{E}$ & (Bergeard et al., 2011) & I & (Ayvazyan, V. et al., 2006) \\
\hline $\mathrm{E}^{\prime}, \mathrm{E}^{\prime \prime}$ & (Holldack et al., 2014) & $\mathrm{J}, \mathrm{K}, \mathrm{J} ', \mathrm{~K}$, & (Lorek et al., 2014) \\
\hline $\mathrm{F}$ & $\mathrm{F}^{\S}$ & $6 \mathrm{~L}, \mathrm{~L}$ & (Leitner et al., 2011) \\
\hline G & (Galayda et al., 2010) & & \\
\hline
\end{tabular}

Table 1. References of the symbols shown in Fig. 2.

${ }^{\dagger}$ Obtained from a survey on the declared photon flux values of a large number of Phtoemission beamlines as made possible by websites of coordinated access projects such as http://wayforlight.eu/eng/search-beamlines.aspx. Measurements performed by the authors on APE-LE beamline (A') confirm part of this range.

${ }^{\ddagger}$ As obtained with a commercial e-gun on measurements performed by the authors.

${ }^{\S}$ From typical performance of a high intensity femtosecond Ti:Sa laser combined with a $10 \%$ efficiency $4 \mathrm{HG}$ stage. 
In Fig. 2 it is also shown (as a dashed black line) the threshold below which the intensity fluctuations are not measurable, as they are smaller than the counting statistics for each pulse (or second):

$$
\gamma=\frac{1}{\sqrt{N}}
$$

for values of $\gamma$ smaller than $1 / \sqrt{N}$, the amount of electron in each pulse is large enough to make the Poisson uncertainty on counting statistics dominating over the intensity fluctuation noise.

The most relevant is the space charge effect. For most of the photoelectron spectroscopies (especially ARPES), the present limit for spin-integrated measurements is of one electron per pulse per reasonable energy and angle interval. Above this, the spectra suffer reduced resolution and deformations. In this configuration, the statistics for single-shot spin polarization measurements is insufficient for both machines, and several shots have to be counted, making the experiment barely feasible at the typical repetition rates of an FEL.

However, very recent experiments of measurement of the spin polarization of the secondary electrons (Fognini et al., 2014) have demonstrated feasibility of experiments with more than $10^{6}$ electrons per pulse, although with reduced polarization signal. The compromise between reduction of the signal and reduction of the uncertainty will probably have to be addressed practically, depending on the aim of the experiment.

On the other hand, the 1CDs higher FOM allows themto outperform significantly the 2CDs when lower intensity, but higher stability sources are considered. ARPES is therefore more immediately applied to this configuration, and it has already been done (Okuda et al., 2011; Bigi, 2016). Yet, also these configurations are extremely photon-hungry, and the ARPES community would surely benefit if the full potential of FEL brilliance was to be unlocked. There is therefore an on-going effort in trying to overcome this issue that has been addressed in (Hellmann et al., 2009; Schönhense et al., 2015; Verna et al., 2016).

Nonetheless, our statistical framework will have to be addressed every time one uses a source of intensity sufficient to achieve $\gamma>\frac{1}{\sqrt{N}}$, independently on how the issue of space charge is addressed, may it be with strong accelerating fields close to the sample(Schönhense et al., 2015), a posteriori data treatment (Schönhense et al., 2015; DellAngela et al., 2015), or exploiting complex time-structures of the pulsed beam (Hellmann et al., 2012). The method is also applicable to any kind of spin-resolved photoelectron spectroscopy from energy integrated measurement of the polarization of the secondary electron yield, to the single channel spin-ARPES, and even to the parallel acquisition spin-ARPES that is now being addressed in different ways (Schönhense et al., 2015; Strocov et al., 2015), as only the number of electron entering the IUCr macros version 2.1.10: 2016/01/28 
polarimeter is relevant for the discussion.

When the vectorial determination of SP is sought the above analysis has to be applied to the three components of SP. Here the 2CDs are "upgraded" easily to 4CDs allowing the same measurement time to provide both the transversal components of SP of the analysed electron beam. Only the third component requires a subsequent measurement act. In the case of Mott polarimeters this can be performed either by alternatively addressing two identical but orthogonal polarimeters by deviating electrostatically the electron beam every other pulse to one or the other of the polarimeters, or by "rotating" the SP of the incoming beam by a magnetic rotator and observe scattering in the same polarimeter. Also when using $1 \mathrm{CD}$ exchange polarimeters two orthogonal apparata are needed and a sequence of eight measurements steps are needed (four magnetization orientations of each of two targets with their normal direction at right angles with each other). In this case an internal calibration can be exploited as one SP component is measured twice (once in each polarimeter).Therefore, when the measurement of the spin polarization vector will require at least two separate measurement steps to a $4 \mathrm{CD}$, while at least 6 measurement steps to a $1 \mathrm{CD}$. Besides the factor of three in the number of measurement steps, $\gamma$ will appear once for each component in the $1 \mathrm{CD}$, as the measurement steps are all temporally interdependent in pairs. In the $4 \mathrm{CD}$, instead, $\gamma$ will never appear, as the measurement steps are all temporally independent from each other.

\section{Numerical experiments}

Numerical Experiments have been performed in order to simulate the two considered experimental setups of the measurements, i.e. the $2 \mathrm{CD}$ and the $1 \mathrm{CD}$ apparata, and to perform a statistically reliable set of simulations to validate the theoretical results discussed above. The numerical code has been written in Mathematica programming language. We sketch, for clarity, a list of the main sets of operations adopted in the numerical simulation plan, in which we tried to follow the true experimental procedure.

The simulation of experiments with 2CD detectors was developed in the following steps:

1. We imagined the true polarization of the electron beam, photoemitted from the sample, $P^{\text {true }}$, to be fixed, since it is an intrinsic property of the physical system.

2. We fixed the known physical parameters of the apparatus, i.e. $\bar{N}_{0}, S_{\text {eff }}, \sigma_{0}, \varepsilon_{2 C D(1 C D)}$.

3. We started a loop over the number of measurement steps, $i$, from 1 to a certain maximum value $n_{p}^{\max }$, in which, for each iteration: 
(a) We randomly extracted with Gaussian probability, representing the distribution of the number of photoemitted electrons (centred in $\bar{N}_{0}$, and with a std. dev. of $\sigma_{0}$ ), the specific number of PE electrons of the $i$-th iteration, $N_{0}(i)$.

(b) From $N_{0}(i)$ we calculated the two quantities

$$
N_{l(r)}^{0}(i)=\text { Round }\left[\left(\frac{1 \pm P^{\text {true }} S_{\text {eff }}}{2}\right) N_{0}(i) \frac{\varepsilon_{2 C D}}{S_{\text {eff }}^{2}}\right]
$$

where with "Round" we indicate the approximation to the nearest integer value.

(c) We then used $N_{l}^{0}(i)$ and $N_{r}^{0}(i)$ as the two mean values of two Poissonians, and then randomly extracted two values, $N_{l}(i)$ and $N_{r}(i)$. This is done to resemble the Poissonian behaviour of the detectors.

(d) The polarization actually detected is thus calculated as

$$
P^{\operatorname{det}}(i)=\frac{N_{l}(i)-N_{r}(i)}{N_{l}(i)+N_{r}(i)} \frac{1}{S_{\text {eff }}}
$$

4. With the array $P^{d e t}\left(i=1: n_{p}^{\max }\right)$ of the detected polarization, we calculated the 'experimental' polarization $P\left(n_{p}\right)$ as a function of the number of measurement steps as the average of the $P^{\text {det }}(i=$ $\left.1: n_{p}^{\max }\right) \mathrm{s}$ up to $n_{p}$ :

$$
P\left(n_{p}\right)=\frac{1}{n_{p}} \sum_{i=1}^{n_{p}} P^{\text {det }}(i), \quad n_{p}=1, \ldots, n_{p}^{\max }
$$

5. Finally, we defined a variance $\Delta P\left(n_{p}\right)$ from the true value $P^{\text {true }}$ as

$$
\Delta P\left(n_{p}\right)=\sqrt{\left(P\left(n_{p}\right)-P^{\text {true }}\right)^{2}}, \quad n_{p}=1, \ldots, n_{p}^{\max }
$$

Each of these simulations, however, is not sufficient to give a significant comparison to Eq. 20 and Eq. 33, because the former are expressed in the statistical limit, i.e. for an infinite number of experiments at each value of $n_{p}$. Therefore, a loop repeating each simulation $n_{\text {sim }}$ times was devised, and the resulting values for each $n_{p}$ were averaged. If $n_{s i m}$ is large enough to be treatable in the statistical limit, the results can then be compared to Eq. 20 and Eq. 33.

For 1CD detectors the procedure was slightly different. We list below the main differences:

1. Each iteration represents a single measurement of electron SP and thus requires two independent measurement steps. Thus the iteration index $j$ does not coincide any more with the number of measurement steps, $i$. The $j$-th measurement is then simulated with two independent extractions (labelled 
as $N_{A}^{0}(j)$ and $\left.N_{B}^{0}(j)\right)$ over the PE electron Gaussian probability distribution. The first extraction is used to calculate $N_{\uparrow}^{0}(j)$ as:

$$
N_{\uparrow \downarrow}^{0}(j)=\text { Round }\left[\left(\frac{1+P^{\text {true }} S_{\text {eff }}}{2}\right) N_{A,(B)}^{0}(j) \frac{\varepsilon_{1 C D}}{S_{\text {eff }}^{2}}\right]
$$

Analogously to $2 \mathrm{CD}$ system, $N_{\uparrow}^{0}(j)$ and $N_{\downarrow}^{0}(j)$ are then used as centres of Poissonian distributions from which the measured values $N_{\uparrow}(j)$ and $N_{\downarrow}(j)$ are extracted. The polarization is then evaluated as:

$$
P^{\operatorname{det}}(j)=\frac{N_{\uparrow}(j)-N_{\downarrow}(j)}{N_{\uparrow}(j)+N_{\downarrow}(j)} \frac{1}{S_{\mathrm{eff}}}
$$

and the variance is determined accordingly.

2. It must be stressed that for $1 \mathrm{CD}$ the use of the same number of measurement steps leads to half the number of measurements with respect to the $2 \mathrm{CD}$. Therefore when average values, as in Eq. (37) and Eq. (38), have to be computed, the summation boundaries must be modified, so $P\left(n_{m}\right)$ and $\Delta P\left(n_{m}\right)$ after $n_{m}$ measurements now read as:

$$
\begin{gathered}
P\left(n_{m}\right)=\frac{1}{n_{m}} \sum_{j=1}^{n_{m}} P^{\text {det }}(j), \\
\Delta P\left(n_{m}\right)=\sqrt{\left(P\left(n_{m}\right)-P^{\text {true }}\right)^{2}},
\end{gathered}
$$

with $n_{m}=1, \ldots, n_{p}^{\max } / 2$.

In order to check the validity of the statistical analysis discussed in Sect. 5 and the necessary approximations, the values of the variance were also calculated by direct substitution of the parameters in Eq. 19 and 31. The results are plotted in Fig. 3 and Fig. 4. The difference between the curves obtained from Eq. 19, 31 and Eq. 20 and 33 in Fig. 4 shows the effects of the approximations of large $n_{p}$. The simulated data show a variance that is always smaller than the statistical estimate, in agreement with the fact that error analysis should give a safe (i.e. as tight as possible, but by excess) evaluation of the experimental uncertainty. 


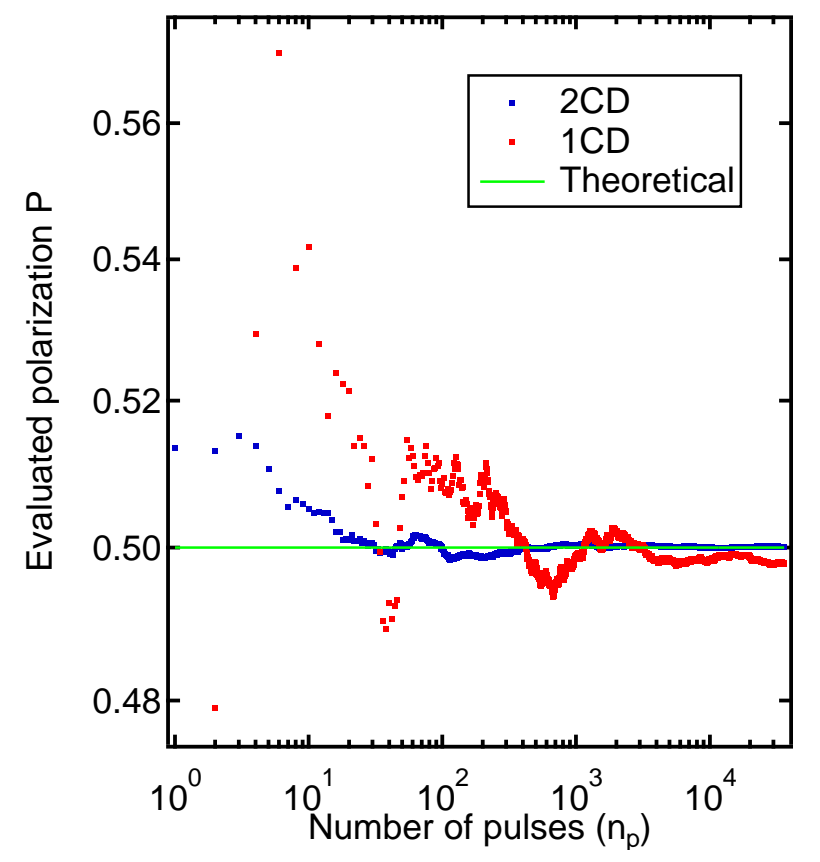

Fig. 3. The result of measurement of a $50 \%$ polarization $\left(P^{\text {true }}=0.5\right)$ with a $1 \mathrm{CD}$ (red) and $2 \mathrm{CD}$ (blue) as a function of the number of averaged pulses.

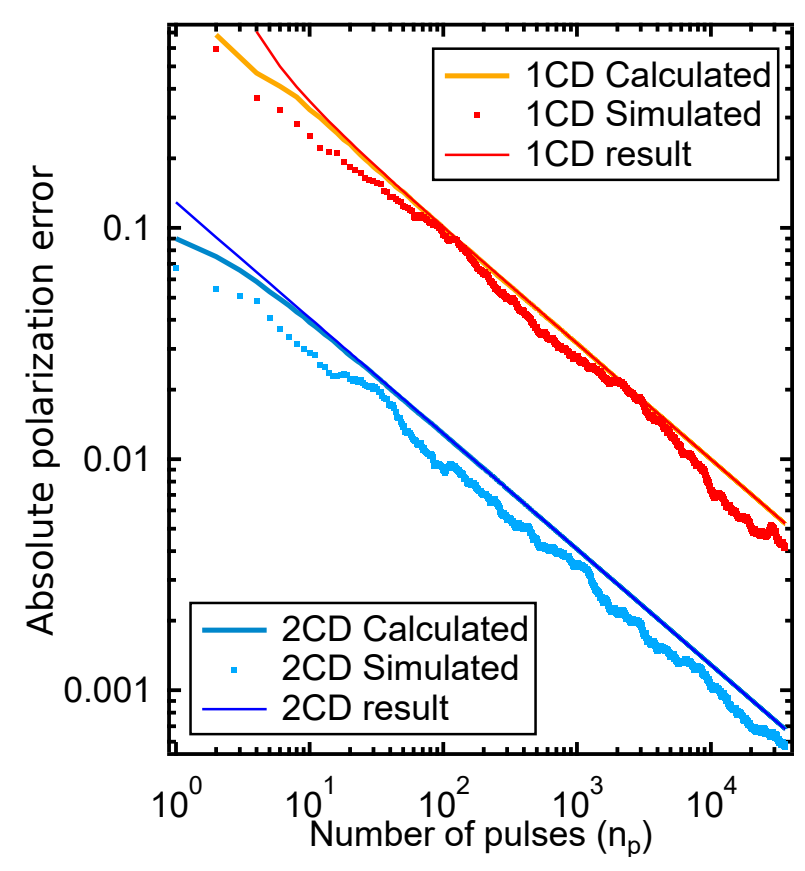

Fig. 4. The results of a simulated run on $1 \mathrm{CD}$ and $2 \mathrm{CD}$ detector. In this case, we used $n_{p}=36000$, corresponding to $1 \mathrm{~h}$ acquisition at $10 \mathrm{~Hz}, \bar{N}_{0}=100000, \sigma_{0}=0.1, S_{\mathrm{eff}}=0.36$ for $1 \mathrm{CD}, \varepsilon_{M}=6 \times 10^{-4}$, $\varepsilon_{V}=1 \times 10^{-2}, N_{\text {sim }}=100$. The solid red and dark blue line show the direct calculation form Eq. 19, 31 , the light blue and orange the results of our approximation for large $n_{p}$, and the dots the simulated measurements. 


\section{Conclusions}

We can therefore conclude that, relying on a solid statistical analysis, we demonstrated that in the pulsed source regime the FOM and effective Sherman function parameters are not comprehensive in describing the detector performance. Instead, the effect of the measurement routine and its interplay with the source time structure must be carefully accounted for.

Despite the lower FOM, indeed, parallel acquisition of asymmetry from two channels (or more) at once decouples the detector from the intensity variations of the source, allowing it to significantly outdo in terms of reduction of statistical error in SP measurement as a function of the averaged number of measurement steps. To prove this, we developed a rigorous description of statistical error for both kinds of detectors, obtaining simple and effective formulas that will be fundamental in the data analysis of this new technique. Finally, we verified such analysis with computational simulations that demonstrated the reliability of our conclusions.

We then mapped out the ranges (and consequently the state of the art sources) in which each detector geometry performs best, individuating three regimes. In the medium-low intensity, small fluctuation regime typical of Synchrotron and continuous sources, the high-FOM 1CDs are superior. In the high brilliance, large shot-to-shot fluctuation pulsed regime characteristic of FEL or high power solid state laser sources, the 2CDs give the best statistics. Finally, in the regime of operation of HHG sources, with low photon flux, high shot-to-shot fluctuations, the performance of $1 \mathrm{CDs}$ and 2CDs is very close.

As time-resolved SP measurements are becoming ubiquitous in advanced spectroscopy of magnetic as well as non-magnetic strongly correlated systems, including spin-textured topological surfaces, and their dynamics needs to be studied, our analysis will be useful to guide the design and the data analysis of such experiments.

\section{Appendix A \\ Calculation details}

In this Section, we expose the details of the calculation concerning the absolute error $\Delta N_{\mathrm{pp}}$ on the difference $N_{0}(i) \sigma_{\uparrow}-N_{0}(i+1) \sigma_{\downarrow}$. Since $N_{0}(i)$ and $N_{0}(i+1)$ are two successive extractions of a normally distributed variable, it is possible to calculate $\Delta N_{\mathrm{pp}}$ as (for convenience of notation we set $N_{0}(i)=x$ and 
$N_{0}(i+1)=y$ and simplify the normalization factors):

$$
\Delta N_{\mathrm{pp}}^{2}=\frac{\int_{0}^{\infty} \int_{0}^{\infty}\left(x \sigma_{\uparrow}-y \sigma_{\downarrow}\right)^{2} e^{-\frac{\left(x-\bar{N}_{0}\right)^{2}}{2 \sigma_{0}^{2}}} e^{-\frac{\left(y-\bar{N}_{0}\right)^{2}}{2 \sigma_{0}^{2}}} \mathrm{~d} x \mathrm{~d} y}{\int_{0}^{\infty} \int_{0}^{\infty} e^{-\frac{\left(x-\bar{N}_{0}\right)^{2}}{2 \sigma_{0}^{2}}} e^{-\frac{\left(y-\bar{N}_{0}\right)^{2}}{2 \sigma_{0}^{2}}} \mathrm{~d} x \mathrm{~d} y}
$$

The lower integration limit can be extended to $-\infty$ because the central value $\bar{N}_{0}$ is positive and large. In this way we can use the known Gaussian integrals (Gradshteyn \& Ryzhik, 2014) that give:

$$
\Delta N_{\mathrm{pp}}^{2}=\bar{N}_{0}^{2}\left(\sigma_{\uparrow}-\sigma_{\downarrow}\right)^{2}+\sigma_{0}^{2}\left(\sigma_{\uparrow}^{2}+\sigma_{\downarrow}^{2}\right)
$$

hence:

$$
\Delta N_{\mathrm{pp}}^{2}=\bar{N}_{0}^{2}\left(\sigma_{\uparrow}^{2}+\sigma_{\downarrow}^{2}\right)\left(\frac{\left(\sigma_{\uparrow}-\sigma_{\downarrow}\right)^{2}}{\sigma_{\uparrow}^{2}+\sigma_{\downarrow}^{2}}+\gamma^{2}\right)
$$

where the aforementioned $\gamma$ has been inserted. The first summand in the last term is small, and can be neglected, giving:

$$
\begin{aligned}
& \Delta N_{\mathrm{pp}}^{2}=\bar{N}_{0}^{2}\left(\sigma_{\uparrow}^{2}+\sigma_{\downarrow}^{2}\right) \gamma^{2}= \\
& =\left(\bar{N}_{\uparrow}^{2}+\bar{N}_{\downarrow}^{2}\right) \gamma^{2} \approx \frac{1}{2}\left(\bar{N}_{\uparrow}+\bar{N}_{\downarrow}\right)^{2} \gamma^{2}
\end{aligned}
$$

As $\bar{N}_{\uparrow} \approx \bar{N}_{\downarrow}$. The result in Eq. (30) of main text is thus justified.

\section{Appendix B \\ Lightsources parameters}

In this section we list the detailed characteristics of the various sources considered in Fig. 2 of main text.

\begin{tabular}{|c|c|c|c|c|c|c|c|}
\hline Lett & flux [ph/s] & R.M.S. fluct. [\%] & En. [eV] & durat. [ps] & rep. rate [Hz] & lens fact. & el flux [e/s] \\
\hline A & $10^{9}-10^{13}$ & $0.1-0.5$ & any $^{8}(20)$ & 50 & $500 \times 10^{6}$ & $10^{-5}$ & $10^{3}-10^{7}$ \\
\hline B & $2 \cdot 10^{12}$ & 0.15 & $21(21)$ & - & - & $10^{-5}$ & $10^{6}$ \\
\hline C & $2 \cdot 10^{15}$ & 1 & $7(7)$ & 20 & $80 \times 10^{6}$ & $10^{-5}$ & $10^{7}$ \\
\hline D & - & $0.25-5$ & $500-4000(1300)$ & - & - & $10^{-3}$ & $10^{5}-10^{9}$ \\
\hline E & - & 1 & any (20) & 40 & $1 \times 10^{6}$ & $10^{-5}$ & $2.5 \times 10^{3}$ \\
\hline E' & $10^{6}$ & 1 & any (20) & 0.1 & $6 \times 10^{6}$ & $10^{-3}$ & $10^{2}$ \\
\hline E" & $10^{6}$ & 1 & any (20) & 0.1 & $6 \times 10^{6}$ & $10^{-5}$ & $10^{0}$ \\
\hline
\end{tabular}




\begin{tabular}{|c|c|c|c|c|c|c|c|}
\hline Lett & flux [ph/pul] & R.M.S. fluct. [\%] & En. $[\mathrm{eV}]$ & durat. [ps] & rep. rate $[\mathrm{Hz}]$ & lens fact. & e. flux [e/pul] \\
\hline F & $8 \cdot 10^{14}$ & 5 & 6 & 0.1 & 1000 & $10^{-3}$ & $10^{8}$ \\
\hline G & $10^{14}$ & 25 & $10-60(30)$ & 0.1 & 10 & $10^{-3}$ & $10^{4}-10^{10}$ \\
\hline H & $10^{13}$ & 50 & $50-300(100)$ & $0.04-0.1$ & 10 & $10^{-3}$ & $10^{4}-10^{9}$ \\
\hline I & $10^{13}$ & 60 & $30-300(30)$ & $0.05-0.2$ & $10^{9}$ & $10^{-3}$ & $10^{4}-10^{9}$ \\
\hline J & $10^{8}$ & 25 & $10-90(30)$ & 0.3 & $20 \times 10^{3}$ & $10^{-3}$ & $10^{4}$ \\
\hline J' & $10^{8}$ & 25 & $10-90(30)$ & 0.3 & $20 \times 10^{3}$ & $10^{-5}$ & $10^{2}$ \\
\hline K & $10^{7}$ & 25 & $10-90(30)$ & 0.3 & $100 \times 10^{3}$ & $10^{-3}$ & $10^{3}$ \\
\hline K' & $10^{7}$ & 25 & $10-90(30)$ & 0.3 & $100 \times 10^{3}$ & $10^{-5}$ & $10^{1}$ \\
\hline L & $10^{6}$ & 26.6 & $10-90(30)$ & 0.3 & $3 \times 10^{3}$ & $10^{-3}$ & $10^{2}$ \\
\hline L' & $10^{6}$ & 26.6 & $10-90(30)$ & 0.3 & $3 \times 10^{3}$ & $10^{-5}$ & $10^{0}$ \\
\hline
\end{tabular}

Acknowledgements The authors acknowledge Dott. G. Panaccione for the fruitful discussions. Funding from the PIK project (MIUR and Sincrotrone Trieste) ULTRASPIN is acknowledged. This work has been partly supported by the NFFA-MIUR project (www.trieste.nffa.eu) and by IOM-CNR.

\section{References}

Allaria, E., Appio, R., Badano, L., Barletta, W., Bassanese, S., Biedron, S., Borga, A., Busetto, E., Castronovo, D., Cinquegrana, P. et al. (2012). Nature Photonics, 6(10), 699-704.

Allaria, E., Castronovo, D., Cinquegrana, P., Craievich, P., Dal Forno, M., Danailov, M., D'Auria, G., Demidovich, A., De Ninno, G., Di Mitri, S. et al. (2013). Nature Photonics, 7(11), 913-918.

AmannJ., BergW., BlankV., DeckerF.-J., DingY., EmmaP., FengY., FrischJ., FritzD., HastingsJ., HuangZ., KrzywinskiJ., LindbergR., LoosH., LutmanA., NuhnH.-D., RatnerD., RzepielaJ., ShuD., Shvyd'koYu., SpampinatiS., StoupinS., TerentyevS., TrakhtenbergE., WalzD., WelchJ., WuJ., ZholentsA. \& ZhuD. (2012). Nat Photon, 6(10), 693-698.

Ayvazyan, V., Baboi, N., Bähr, J. et al. (2006). Eur. Phys. J. D, 37(2), 297-303.

Bergeard, N., Silly, M. G., Krizmancic, D., Chauvet, C., Guzzo, M., Ricaud, J. P., Izquierdo, M., Stebel, L., Pittana, P., Sergo, R., Cautero, G., Dufour, G., Rochet, F. \& Sirotti, F. (2011). Journal of Synchrotron Radiation, 18(2), $245-250$.

Bertacco, R. \& Ciccacci, F. (1999). Surface science, 419(2), 265-271.

Bertacco, R., Merano, M. \& Ciccacci, F. (1998). Applied Physics Letters, 72(16), 2050-2052.

Bigi, C. (2016). Complete photoemission experiment for probing spin texture at surfaces: commissioning of VLEED detectors for 3D spin-resolved ARPES and first experiments. Master's thesis, Università degli studi di Milano.

Burnett, G. C., Monroe, T. J. \& Dunning, F. B. (1994). Review of Scientific Instruments, 65(6), 1893-1896.

Das, P. K., Di Sante, D., Vobornik, I., Fujii, J., Okuda, T., Bruyer, E., Gyenis, A., Feldman, B. E., Tao, J., Ciancio, R., Rossi, G., Ali, M. N., Picozzi, S., Yadzani, A., Panaccione, G. \& Cava, R. J. (2016). Nat Commun, 7. Article.

DellAngela, M., Anniyev, T., Beye, M., Coffee, R., A., F., Gladh, J., Kaya, S., Katayama, T., Krupin, O., Nilsson, A., Nordlund, D., Schlotter, W. F., Sellberg, J. A., Sorgenfrei, F., Turner, J. J., Öström, H., Ogasawara, H., Wolf, M. \& Wurth, W. (2015). Structural Dynamics, 2(2).

Dil, J. H. (2009). Journal of Physics: Condensed Matter, 21(40), 403001.

Fognini, A., Salvatella, G., Michlmayr, T., Wetli, C., Ramsperger, U., Bähler, T., Sorgenfrei, F., Beye, M., Eschenlohr, A., Pontius, N. et al. (2014). New Journal of Physics, 16(4), 043031.

Galayda, J. N., Arthur, J., Ratner, D. F. \& White, W. E. (2010). J. Opt. Soc. Am. B, 27(11), B106-B118.

Gay, T. J. \& Dunning, F. B. (1992). Review of Scientific Instruments, 63(2), 1635-1651.

Gay, T. J., Khakoo, M., Brand, J., Furst, J., Meyer, W., Wijayaratna, W. \& Dunning, F. (1992). Review of scientific instruments, 63(1), 114-130.

Getzlaff, M., Heidemann, B., Bansmann, J., Westphal, C. \& Schönhense, G. (1998). Review of Scientific Instruments, 69(11), 3913-3923.

Gradshteyn, I. S. \& Ryzhik, I. M. (2014). Table of Integrals, Series, and Products. Elsevier Science. 
Graf, J., Jozwiak, C., Schmid, A. K., Hussain, Z. \& Lanzara, A. (2005). Phys. Rev. B, 71, 144429.

Hellmann, S., Rossnagel, K., Marczynski-Buhlow, M. \& Kipp, L. (2009). Phys. Rev. B, 79, 035402.

Hellmann, S., Sohrt, C., Beye, M., Rohwer, T., Sorgenfrei, F., Marczynski-Bühlow, M., Kalläne, M., Redlin, H., Hennies, F., Bauer, M., Föhlisch, A., Kipp, L., Wurth, W. \& Rossnagel, K. (2012). New Journal of Physics, 14(1), 013062.

Hoesch, M., Greber, T., Petrov, V., Muntwiler, M., Hengsberger, M., Auwärter, W. \& Osterwalder, J. (2002). Journal of Electron Spectroscopy and Related Phenomena, 124(2-3), 263 - 279. Frontiers in photoemission spectroscopy of solids and surfaces.

Holldack, K., Bahrdt, J., Balzer, A., Bovensiepen, U., Brzhezinskaya, M., Erko, A., Eschenlohr, A., Follath, R., Firsov, A., Frentrup, W., Le Guyader, L., Kachel, T., Kuske, P., Mitzner, R., Müller, R., Pontius, N., Quast, T., Radu, I., Schmidt, J.-S., Schüßler-Langeheine, C., Sperling, M., Stamm, C., Trabant, C. \& Föhlisch, A. (2014). Journal of Synchrotron Radiation, 21(5), 1090-1104.

Kessler, J. (1985). Polarized electrons. Springer-Verlag Berlin Heidelberg GmbH.

Kirschner, J., Giebels, F., Gollisch, H. \& Feder, R. (2013). Physical Review B, 88(12), 125419.

Kutnyakhov, D., Lushchyk, P., Fognini, A., Perriard, D., Kolbe, M., Medjanik, K., Fedchenko, E., Nepijko, S., Elmers, H., Salvatella, G., Stieger, C., Gort, R., Bähler, T., Michlmayer, T., Acremann, Y., Vaterlaus, A., Giebels, F., Gollisch, H., Feder, R., Tusche, C., Krasyuk, A., Kirschner, J. \& Schönhense, G. (2013a). Ultramicroscopy, 130, 63 - 69. Eighth International Workshop on LEEM/PEEM.

Kutnyakhov, D., Lushchyk, P., Fognini, A., Perriard, D., Kolbe, M., Medjanik, K., Fedchenko, E., Nepijko, S., Elmers, H., Salvatella, G., Stieger, C., Gort, R., Bähler, T., Michlmayer, T., Acremann, Y., Vaterlaus, A., Giebels, F., Gollisch, H., Feder, R., Tusche, C., Krasyuk, A., Kirschner, J. \& Schönhense, G. (2013b). Ultramicroscopy, 130(0), 63 - 69. Eighth International Workshop on LEEM/PEEM.

Leitner, T., Sorokin, A. A., Gaudin, J., Kaser, H., Kroth, U., Tiedtke, K., Richter, M. \& Wernet, P. (2011). New Journal of Physics, 13(9), 093003.

Lorek, E., Larsen, E. W., Heyl, C. M., Carlström, S., Paleček, D., Zigmantas, D. \& Mauritsson, J. (2014). Review of Scientific Instruments, 85(12).

Mott, N. F. (1929). Proceedings of the Royal Society of London. Series A, 124(794), 425-442.

Mott, N. F. (1932). Proceedings of the Royal Society of London. Series A, 135(827), 429-458.

Okuda, T., Miyamaoto, K., Miyahara, H., Kuroda, K., Kimura, A., Namatame, H. \& Taniguchi, M. (2011). Review of Scientific Instruments, 82(10), -.

Petrov, V. N., Grebenshikov, V. V., Andronov, A. N., Gabdullin, P. G. \& Maslevtcov, A. V. (2007). Review of Scientific Instruments, $\mathbf{7 8}(2),-$.

Petrov, V. N., Grebenshikov, V. V., Grachev, B. D. \& Kamochkin, A. S. (2003). Review of Scientific Instruments, $\mathbf{7 4}(3), 1278-1281$.

Petrov, V. N. \& Kamochkin, A. S. (2004). Review of Scientific Instruments, 75(5), 1274-1279.

Pincelli, T., Petrov, V. N., Brajnik, G., Ciprian, R., Lollobrigida, V., Torelli, P., Krizmancic, D., Salvador, F., Luisa, A. D., Sergo, R., Gubertini, A., Cautero, G., Carrato, S., Rossi, G. \& Panaccione, G. (2016). Rev. Sci. Instrum. $\mathbf{8 7}(3)$, In press.

Schönhense, G., Medjanik, K. \& Elmersl, H.-J. (2015). Journal of Electron Spectroscopy and Related Phenomena, 200, 94.

Sherman, N. (1956). Phys. Rev. 103, 1601-1607.

Shimojima, T., Okazaki, K. \& Shin, S. (2015). Journal of the Physical Society of Japan, 84(7), 072001.

Shull, C. G., Chase, C. T. \& Myers, F. E. (1943). Phys. Rev. 63, 29-37.

Strocov, V. N., Petrov, V. N. \& Dil, J. H. (2015). Journal of Synchrotron Radiation, 22(3), 708.

Suzuki, R., Sakano, M., Zhang, Y., Akashi, R., Morikawa, D., Harasawa, A., Yaji, K., Kuroda, K., Miyamoto, K., Okuda, T. et al. (2014). Nature nanotechnology, 9(8), 611-617.

Toru Hara, Kazuaki Togawa, H. T. (2012). In Proceedings of FEL2012, Nara, Japan, edited by V. R. S. Toshinari Tanaka, pp. 5-8. JACoW.

Tusche, C., Ellguth, M., Krasyuk, A., Winkelmann, A., Kutnyakhov, D., Lushchyk, P., Medjanik, K., Schönhense, G. \& Kirschner, J. (2013). Ultramicroscopy, 130(0), 70 - 76. Eighth International Workshop on LEEM/PEEM.

Verna, A., Greco, G., Lollobrigida, V., Offi, F. \& Stefani, G. (2016). Journal of Electron Spectroscopy and Related Phenomena, 209, $14-25$.

Yu, D., Math, C., Meier, M., Escher, M., Rangelov, G. \& Donath, M. (2007). Surface Science, 601(24), 5803 - 5808. Wagga Symposium on Surfaces and Interfaces - 2006 Wagga Symposium on Surfaces and Interfaces - 2006.

\section{Synopsis}

In this work we theoretically derive the experimental uncertainties concerning electron spin polarization (SP) in various realistic measurement conditions. Supported by numerical simulations, our results define new mathematical instruments for handling the correct statistics of SP measurements in presence of the source intensity fluctuations and are applied to the characteristics of a wide set of state-state-of-the-art facilities.

IUCr macros version 2.1.10: 2016/01/28 